\title{
'I don't have to go to the gym because I ate very healthy today': the development of a scale to assess diet-related compensatory health beliefs
}

\author{
Maartie P Poelman*, Willemijn M Vermeer, Ellis L Vyth and Ingrid HM Steenhuis \\ Department of Health Sciences and the EMGO Institute for Health and Care Research, VU University, \\ De Boelelaan 1085, 1081 HV Amsterdam, The Netherlands
}

Submitted 31 May 2011: Final revision received 3 April 2012: Accepted 13 April 2012: First published online 22 May 2012

\begin{abstract}
Objective: Nutritional interventions to decrease energy intake, aimed at portion sizes and front-of-package labelling, are effective only if people do not compensate for their reduced energy intake. Since several observational studies indicate that these interventions could prompt compensation behaviour, it is important to assess underlying beliefs. Therefore, the purpose of the two studies reported here was to develop a Diet-related Compensatory Health Beliefs Scale (Diet-CHBS).

Design: Cross-sectional surveys were conducted for the scale development. Study 1 provided data on the factor analysis and convergent validity, while Study 2 assessed the Diet-CHBS' test-retest reliability.

Settings: VU University Amsterdam (Study 1) and twenty-five worksite cafeterias in the Netherlands (Study 2).

Subjects: Study 1 was conducted among 179 students and their parents; Study 2 was conducted among 119 worksite cafeteria visitors.

Results: The results of Study 1 showed that the scale consisted of the hypothesized factors of compensation beliefs with regard to portion sizes $(\alpha=0.73)$, front-of-package health logos $(\alpha=0.77)$ and exercise $(\alpha=0.75)$. The scale's overall Cronbach's $\alpha$ was $0 \cdot 82$. The Diet-CHBS had a Pearson correlation of $0 \cdot 32$ with a general health compensatory beliefs scale, signifying satisfactory convergent validity. Study 2 showed that the intra-class correlation coefficient between T1 and T2 was $0 \cdot 69$, indicating adequate test-retest reliability.

Conclusion: The Diet-CHBS is a valid and reliable instrument for assessing dietrelated compensatory health beliefs in response to nutritional interventions. It is important to take such beliefs into account in further intervention studies aimed at preventing overweight and obesity.
\end{abstract}

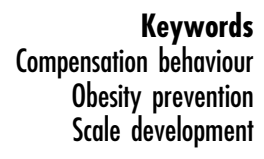

In response to the increasing prevalence of overweight and obesity and its associated consequences, several nutritional interventions have been developed aimed at reducing energy intake ${ }^{(1,2)}$. Nutritional interventions aimed at reducing energy intake can have a quantitative dimension (reducing the overall amount of foods eaten) or a qualitative dimension (stimulating the intake of products with a more favourable nutrient composition) ${ }^{(3-5)}$.

Despite these interventions to reduce people's energy intake, overweight and obesity prevalence is not decreasing $^{(6,7)}$. Although several factors and individual differences contribute to this problem, part of the explanation might be found in the fact that people compensate in response to nutritional interventions. That is, people compensate for the reduced energy intake that was accomplished by the intervention with other behaviour before or afterwards (e.g. when people believe that it is not necessary to go to the gym because they already ate healthy foods or they believe they can eat a larger amount of healthier variants of snacks compared with the unhealthier variants). Consequently, the intervention effect is neutralized (e.g. when people would burn an equal amount of energy if they went to the gym instead of eating healthier as a result of the nutritional intervention) or the nutritional intervention results in an even more positive energy balance because of compensating (e.g. when people would have a lower energy intake if they ate just one unhealthy snack instead of more - but healthier - snacks).

Several experimental studies have shown that nutritional interventions aimed at promoting products with a more favourable nutritional composition by means of a so-called 'front-of-package label' could provoke compensation behaviour. Front-of-package labels are labels or logos on the front of a food package that represent a 
simplified and overall representation of the main nutrient composition and can define the 'healthiness' of products within a certain food category ${ }^{(8)}$. Furthermore, experimental studies have indicated that interventions which stimulate the intake of smaller food portions also might elicit compensation behaviour. For example, studies showed that when products were healthy, or described as healthier, people ate more of these products compared with the unhealthy variants ${ }^{(9,10)}$. Another study revealed that people also tend to systematically underestimate the energy content of healthy food ${ }^{(11)}$. In addition, a study into diet foods showed that the consumption of such products encouraged people to overeat ${ }^{(12)}$. Moreover, a study in which different plate sizes were offered during a buffet showed that the people with the smallest plates ate the same amount of food as those who had the biggest plates. They compensated for their small plate size by walking to the buffet more often ${ }^{(13)}$.

Additionally, studies in the area of the 'licensing effect' reveal the likelihood of energy balance compensation behaviour. The licensing effect refers to people's perceived license to engage in indulgent behaviour after engaging in positive behaviour ${ }^{(14)}$. A previous study demonstrated that individuals who sampled an item framed as healthy consumed more than those who sampled an item framed as tasty. This outcome counted in particular for participants who were less concerned about their weight ${ }^{(15)}$. Another study into licensing effects as a consequence of the use of dietary supplements showed that participants who took what they believed to be a dietary supplement (actually a placebo) revealed less desire for exercise, more desire for hedonic activities and were more likely to prefer the buffet over an organic meal ${ }^{(16)}$.

Although these studies indicate the presence of compensation behaviour, none of them has revealed the rationale behind compensation behaviour. In order to get more insight into the drive behind behaviour, it is essential to assess beliefs. Understanding beliefs can be helpful in the development and evaluation of interventions. For example, health educational programmes can be developed to shape people's beliefs or make them aware about the ineffectual consequences of compensation behaviour in reaction to nutritional interventions. Additionally, in effect evaluations of diet-related health promotion interventions, insight into beliefs can be helpful in understanding study results. Furthermore, the impact of diet-related compensation beliefs on the main outcomes can be determined. In doing so, more comprehensive study results can be presented. Nevertheless, no scale to assess diet-related compensatory health beliefs is yet available.

Therefore, the aim of the two studies reported in the present paper was to develop a valid and reliable questionnaire to assess such diet-related compensation beliefs. The first study describes the development of the scale and provides data on its internal consistency and convergent validity. The objective of the second study was to test the scale's reliability over time.

\section{Study 1: Development of the Diet-CHBS items and testing the scale's internal consistency and validity}

\section{Metbods}

Scale item development

Experts in the field of obesity prevention created the items of the so-called 'Diet-related Compensatory Health Beliefs Scale' (Diet-CHBS). Based on the literature, different aspects of diet-related compensation beliefs were explored and discussed. As mentioned before, previous research indicated that interventions aimed at decreasing energy intake by means of portion sizes or front-of-package labelling could provoke compensation behaviour. Therefore, items about compensation behaviours in reaction to these types of interventions were developed. Furthermore, physical activity was taken into account in the development of the items since it was hypothesized that people could decrease or eliminate physical activity as a means to compensate for nutritional interventions.

Fourteen items were created, all having a 5-point Likert response option ('totally disagree' to 'totally agree'). All items measure compensatory beliefs by which potential positive effects of nutritional interventions can be neutralized by negative effects of other behaviour. Examples of items are 'If I eat a small meal, there is no harm in eating more cookies and candies' and 'When I eat less, it's not necessary to have a lot of exercise'.

\section{Study design and participants}

A cross-sectional survey was conducted among Dutch students ( $n$ 68) and aspiring students and their parents ( $n$ 128). Students were recruited during a second year Health Sciences lecture and the aspiring students and their parents were recruited during an information lecture about studying at VU University Amsterdam. They were asked to complete the questionnaire that was handed out.

The majority of the participants were female $(79 \cdot 1 \%)$ and participants' ages ranged from 17 to 61 years (mean 27 (SD 13.2) years). The prevalence of overweight in this sample was $10 \cdot 4 \%$, whereas $42 \%$ of adult females and $51 \%$ of adult males in the Dutch population are overweight or obese ${ }^{(7)}$.

\section{Measures}

The questionnaire consisted of the fourteen Diet-CHBS items, seventeen items from the compensatory health belief scale (CHBS) ${ }^{(17)}$ and four demographic questions (i.e. date of birth, sex, height and body weight).

The CHBS was included in this questionnaire to validate the Diet-CHBS. The CHBS is a valid and reliable seventeenitem questionnaire which examines beliefs regarding the 
extent to which several types of unhealthy behaviour could compensate for or neutralize healthy behaviour and the other way around (how healthy behaviour could be compensated by unhealthy behaviour). The CHBS contains items such as 'Smoking can be compensated for by exercising' and 'Too little sleep during the week can be compensated for by sleeping in on the weekends ${ }^{,(17)}$. Its Cronbach's $\alpha$ was $0 \cdot 61$.

\section{Statistical methods}

Internal consistency. In order to measure the internal consistency of the Diet-CHBS, an exploratory principal components factor analysis with oblique rotation was used. The items were not allowed to have a correlation above 0.9 and the values of the anti-image matrix had to be higher than 0.5 to be included in the remaining analyses. Further, items were retained in the analysis when the eigenvalues that were associated with each factor were above $1 \cdot 0$. Last, the scale items had to load above 0.4 on one of the factors ${ }^{(18)}$.

Cronbach's $\alpha$ was calculated to test the internal consistency of the Diet-CHBS. Cronbach's $\alpha$ values were analysed for the total scale and for the different factors. Based on criteria set in the literature, a value of Cronbach's $\alpha$ above 0.8 was considered to be an indicator for a good reliability and Cronbach's $\alpha$ above 0.7 was defined as an indicator for an adequate reliability. Factors with Cronbach's $\alpha$ values below $0 \cdot 4$ indicated a low internal consistency of the items related to that factor and were excluded from further analyses ${ }^{(18)}$.

Validation. The aim of the validation was to gather evidence of the convergent validity with reference to the CHBS of Knäuper et al. ${ }^{(17)}$. In order to explore the validity, the relationship between the Diet-CHBS and the CHBS was examined with a Pearson correlation test. It was hypothesized that there would be a positive correlation between them because both scales assess compensatory health beliefs. Additionally, a Pearson correlation test was also conducted between the diet subscale of the CHBS and the solely diet subscales of the Diet-CHBS (Table 3; subscales 2 and 3). The correlation had to be below $0 \cdot 9$.
All data were analysed using the SPSS for Windows statistical software package version $15 \cdot 0$.

\section{Results}

Participants

Of the 196 participants, 179 participants had answered all measures of the questionnaire and were included in the analysis.

\section{Internal consistency}

The fourteen items of the Diet-CHBS were subjected to factor analysis to explore the factor structure. Since most of the created items about diet-related compensatory health beliefs were related to portion sizes, front-of-package labelling and exercise, subscales related to these topics were expected.

The determinant of the correlation matrix $(0 \cdot 11)$, the Kaiser-Meyer-Olkin test of sampling adequacy $(0 \cdot 80)$ and Bartlett's test of sphericity (780 149, $\mathrm{df}=91, P<0 \cdot 001$ ) showed that the data were suitable for factor analysis.

Four factors with an eigenvalue above 1.0 were extracted, which was similar to the scree plot that also showed four factors. Nevertheless, the eigenvalue of the fourth factor, containing three items that were all about compensation behaviour related to eating unhealthy snacks, was only $1 \cdot 10$. Further, this factor had a Cronbach's $\alpha$ of 0.36 which was below $0 \cdot 4$. Therefore, the fourth factor was excluded and only the first three factors, with a total of eleven items, were included in further analyses.

Factor extraction was repeated on the remaining eleven items. Oblique rotation (direct oblimin) was used because it was assumed that the items of the different factors were interrelated to some degree. The correlation matrix (Table 1 ) showed that the items did not correlate above $0 \cdot 9$ and the values of the anti-image matrix were all above 0.5 . The inter-class correlations of the Diet-CHBS showed that the factors were interrelated (Table 2). Furthermore, all items had a factor loading above $0 \cdot 4$ (Table 3 ) and therefore no items were deleted. Based on these outcomes, the three factors remained in the scale.

Table 1 Correlation matrix of the eleven items of the Diet-related Compensatory Health Beliefs Scale (Diet-CHBS)

\begin{tabular}{|c|c|c|c|c|c|c|c|c|c|c|c|}
\hline & Q1 & Q2 & Q3 & Q4 & Q5 & Q6 & Q7 & Q8 & Q9 & Q10 & Q11 \\
\hline Q1 & 1.000 & - & - & - & - & - & - & - & - & - & - \\
\hline Q2 & 0.170 & $1 \cdot 000$ & - & - & - & - & - & - & - & - & - \\
\hline Q3 & 0.451 & 0.113 & 1.000 & - & - & - & - & - & - & - & - \\
\hline Q4 & 0.226 & 0.394 & 0.240 & 1.000 & - & - & - & - & - & - & - \\
\hline Q5 & 0.466 & 0.272 & 0.375 & 0.326 & 1.000 & - & - & _- & - & - & - \\
\hline Q6 & $0 \cdot 161$ & 0.196 & 0.416 & 0.075 & 0.280 & 1.000 & - & - & - & - & - \\
\hline Q7 & 0.300 & $0 \cdot 186$ & 0.388 & 0.179 & 0.328 & 0.630 & $1 \cdot 000$ & - & - & - & - \\
\hline Q8 & 0.229 & 0.319 & 0.221 & 0.371 & 0.371 & 0.304 & 0.274 & 1.000 & - & - & - \\
\hline Q9 & 0.345 & $0 \cdot 100$ & 0.560 & 0.185 & 0.516 & 0.477 & 0.465 & 0.359 & 1.000 & - & - \\
\hline Q10 & 0.105 & 0.551 & 0.056 & 0.384 & 0.289 & 0.193 & 0.182 & 0.364 & 0.219 & 1.000 & - \\
\hline Q11 & 0.287 & 0.263 & 0.303 & 0.113 & 0.246 & 0.405 & 0.540 & 0.104 & 0.342 & 0.193 & $1 \cdot 000$ \\
\hline
\end{tabular}

Pearson correlation coefficients between all pairs of the items. All correlation coefficients are below 0.9. 
The first factor (items 1-4, Table 3) contained items that reflected compensatory beliefs related to exercise and had an $\alpha$ of $0 \cdot 75$. The second factor was concerned with compensatory beliefs related to portion sizes (items $5-8$, Table 2) and had an $\alpha$ of $0 \cdot 73$. The third factor reflected compensatory beliefs associated with products with frontof-package logos (items 9-11, Table 3) and had an $\alpha$ of $0 \cdot 77$. The mean scores and standard deviations for each factor were subsequently $2 \cdot 02$ (SD 0.79), $2 \cdot 73$ (SD 0.80) and $2 \cdot 60$ (SD 0.99). The overall Cronbach's $\alpha$ of the DietCHBS was $0 \cdot 82$.

\section{Validation}

To analyse the convergent validity of the scale, the Pearson correlation was measured between the Diet-CHBS and the CHBS. The test showed a significant correlation of 0.32 $(P \leq 0 \cdot 001)$, indicating a positive association between the two scales. Furthermore, the correlation between the

Table 2 Inter-correlations of the three factors of the Diet-related Compensatory Health Beliefs Scale (Diet-CHBS)

\begin{tabular}{lccc}
\hline Factor & 1 & 2 & 3 \\
\hline 1. Exercise & - & & \\
2. Portion size & 0.26 & - & \\
3. Front-of-package labelling & 0.34 & 0.19 & - \\
\hline
\end{tabular}

The inter-correlation matrix indicates the acceptable use of oblique rotation. diet-related items of the CHBS and the diet-related items of the Diet-CHBS (Table 3; subscales 2 and 3) was somewhat higher $(0 \cdot 34, P \leq 0 \cdot 001)$.

\section{Study 2: Test-retest reliability of the Diet-CHBS}

\section{Methods}

\section{Study design and participants}

In order to test the test-retest reliability of the Diet-CHBS, a group of Dutch worksite cafeteria visitors of various companies were included in the study. This population had already participated in a study that assessed the effectiveness of offering worksite cafeteria visitors a small portion of a hot meal in addition to the available size in order to decrease energy intake ${ }^{(19)}$. In the present study, a total of 384 visitors of twenty-five worksite cafeterias received four online questionnaires. For pre-test purposes, these respondents filled in the Diet-CHBS that was part of the fourth online questionnaire. Afterwards, 191 of the initial 384 respondents were willing to participate in the retest. Two weeks later, they received an online version of the Diet-CHBS again. Finally, 119 participants completed the post-test and were included in the test-retest analysis. The majority of these participants were female $(61 \cdot 1 \%)$ and their age ranged from 19 to 63 years (mean 41 (sD 10.9) years). The overweight and obesity prevalence in this sample was $32 \cdot 2 \%$.

Table 3 Cronbach's $\alpha$ values, factor loadings, mean scores and standard deviations of the Diet-related Compensatory Health Beliefs Scale (Diet-CHBS)

\begin{tabular}{|c|c|c|c|c|c|c|}
\hline & Cronbach's $\alpha$ & 1 & 2 & 3 & Mean & SD \\
\hline Diet-CHBS - overall & $0 \cdot 82$ & & & & & \\
\hline $\begin{array}{l}\text { Factor 1: Diet-CHB related to exercise } \\
\text { 1. To maintain your weight, it is fine to have less exercise if you eat small portions } \\
\text { 2. To maintain your weight, it is fine to have less exercise if you eat products with } \\
\text { a front-of-package logo }\end{array}$ & $0 \cdot 75$ & $\begin{array}{l}0.78 \\
0.70\end{array}$ & $\begin{array}{l}0.01 \\
0 \cdot 15\end{array}$ & $\begin{array}{l}0.05 \\
0.25\end{array}$ & $2 \cdot 02$ & 0.79 \\
\hline $\begin{array}{l}\text { 3. When I eat less, it's not necessary to have a lot of exercise } \\
\text { 4. When I mainly eat products with a front-of-package logo, it is not necessary } \\
\text { to have a lot of exercise }\end{array}$ & & $\begin{array}{l}0.68 \\
0.59\end{array}$ & $\begin{array}{l}0.25 \\
0.03\end{array}$ & $\begin{array}{l}0.01 \\
0.37\end{array}$ & & \\
\hline $\begin{array}{l}\text { Factor 2: Diet-CHB related to portion size } \\
5 \text {. If I eat a small meal, it's fine to have a larger portion during the next meal } \\
\text { 6. To maintain your weight, it is fine to eat a large meal, if you eat a small portion } \\
\text { during the next meal }\end{array}$ & 0.73 & $\begin{array}{l}0 \cdot 14 \\
0 \cdot 15\end{array}$ & $\begin{array}{l}0.83 \\
0.82\end{array}$ & $\begin{array}{l}0.12 \\
0.15\end{array}$ & $2 \cdot 73$ & $0 \cdot 80$ \\
\hline $\begin{array}{l}\text { 7. To maintain your weight, it is fine to eat a high caloric snack in between meals, } \\
\text { if you compensate for this by eating a smaller portion later in the day } \\
\text { 8. If I eat a small meal, there is no harm in eating more cookies and candies }\end{array}$ & & $\begin{array}{l}0.31 \\
0.31\end{array}$ & 0.51 & $\begin{array}{l}0.20 \\
0.03\end{array}$ & & \\
\hline $\begin{array}{l}\text { Factor 3: Diet-CHB related to products with a front-of-package logo } \\
\text { 9. When I eat products with a front-of-package logo, I have to take less account } \\
\text { of the amount I eat of these products than when I eat products without a } \\
\text { front-of-package logo }\end{array}$ & $0 \cdot 77$ & 0.04 & 0.03 & 0.81 & $2 \cdot 60$ & 0.99 \\
\hline $\begin{array}{l}\text { 10. When I eat snacks that have a front-of-package logo in between meals, it is fine to } \\
\text { eat more of these products compared with snacks without a front-of-package } \\
\text { logo }\end{array}$ & & $0 \cdot 13$ & 0.03 & 0.79 & & \\
\hline $\begin{array}{l}\text { 11. When I drink dairy products with a front-of-package logo as opposed to dairy } \\
\text { products without a front-of-package logo, it is fine to have more of these } \\
\text { products }\end{array}$ & & 0.02 & 0.08 & 0.74 & & \\
\hline
\end{tabular}

The items of the Diet-CHBS after factor extraction. The second column shows the Cronbach's $\alpha$ value for the overall scale and for the separate subscales. The next three columns show the factor loadings that are from the pattern matrix after oblique rotation. All bold factor loadings are above $0 \cdot 4$ and relate to the described factor. The last two columns show the mean scores and standard deviation of the corresponding factor. 


\section{Statistical methods}

Participants. To be included in the analysis, participants had to fill in all eleven items of the Diet-CHBS at both test moments.

Test-retest analysis. First, the Cronbach's $\alpha$ for the overall scale was measured at both test moments. Further, the test-retest reliability was analysed by measuring the intra-class coefficient (ICC) for single measures between the scores of the two test moments. An ICC of minimal 0.7 indicates that the scale has good test-retest reliability.

\section{Results}

\section{Participants}

Of the initial 384 worksite cafeteria visitors, 119 participants (31\%) completed all items of the Diet-CHBS at both test moments and were included in the test-retest analysis.

\section{Test-retest analysis}

The Cronbach's $\alpha$ values for the Diet-CHBS were 0.79 (T1) and $0 \cdot 81$ (T2), indicating a good internal reliability. The single measure ICC of the Diet-CHBS between T1 and T2 was $0.69(P<0.001)$. This value is almost 0.7 and therefore an adequate test-retest reliability was assumed.

\section{General discussion}

In the present paper, a scale measuring diet-related compensatory health beliefs was developed. The scale's internal consistency, convergent validity and test-retest reliability were assessed. The Diet-CHBS is an eleven-item questionnaire and can be used to determine diet-related compensatory health beliefs in response to nutritional interventions aimed at food portion sizes or products with front-of-package labelling to reduce energy intake. To our best knowledge, the Diet-CHBS is the first scale that focuses specifically on diet-related compensatory health beliefs in response to nutritional interventions. Furthermore, the paper highlights the importance of paying attention to this potentially negative side-effect of nutritional interventions aimed at preventing overweight and obesity.

As hypothesized, factors about compensation beliefs related to exercise, food portions and front-of-package labelling were generated. The first factor comprises beliefs about the idea that if you eat small food portions or products with a front-of-package logo, exercise is not required in order to balance your weight. The second factor comprises beliefs about the idea that you can eat unhealthy snacks or large meals if you want to balance your weight, you just have to eat smaller portions or meals in between. The third factor comprises beliefs about the idea that the amount of foods eaten is of less importance in balancing body weight, as long as the product contains a front-of-package logo.
In addition to these expected factors, a fourth factor was extracted from the analysis. Nevertheless, this factor was excluded from the final Diet-CHBS because of its low eigenvalue and Cronbach's $\alpha$. A viable explanation for the low internal consistency compared with the other factors of the Diet-CHBS is that all items of this factor were snackrelated. It is possible that eating unhealthy snacks is associated with a different type of behaviour, i.e. a more affective - hedonistic - type which is not related to healthy eating or compensation behaviour at all.

As hypothesized, the Diet-CHBS had a significant association with the CHBS because both scales measure compensatory health beliefs ${ }^{(17)}$. Also the diet-related items of the Diet-CHBS showed a significant positive association with the diet-related subscale of the CHBS. Furthermore, the test-retest showed an adequate ICC between $\mathrm{T} 1$ and $\mathrm{T} 2$. These outcomes indicated the validity of the Diet-CHBS and that it is stable over time. Therefore it can be concluded that the scale is a valid instrument with high internal consistency and adequate test-retest reliability.

Although observational studies have indicated that compensation behaviour can be a side-effect of interventions in order to decrease energy intake aimed at portion sizes and front-of-package logos, so far no study has focused specifically on such underlying diet-related compensatory health beliefs. Therefore the present study contributes to the literature by offering new insights into people's eating behaviour when they are faced with nutritional interventions. An additional strength of the study is that two different populations were used to test the internal consistency, convergent validity and test-retest reliability of the Diet-CHBS. Strengths of the scale are the high internal consistency of both the overall scale and the separate subscales. Furthermore, this scale determines a new important area of nutritional behaviour.

The study and Diet-CHBS also have some limitations. The population used to test the scale's internal consistency and validity was quite young and presumably had a high socio-economic status since all participants were recruited at a university. The majority of the participants were female and had a healthy body weight. Since the overweight and obesity prevalence was much lower in this sample as compared with the Dutch population, it appears that this sample was not representative for the whole Dutch population. The difference in BMI is probably the due to the highly educated population used in Study 1. The sample that participated in Study 2 was more representative for the Dutch population.

A limitation of the scale is that the items of the DietCHBS focus only on compensation beliefs associated with interventions related to portion sizes and products with front-of-package logos. There are also other types of nutritional interventions that aim to reduce people's energy intake. For example, stimulating the intake of lean 
products can be a qualitative intervention to decrease people's energy intake. These products are suitable in an energy-restricted diet but do not always contain a frontof-package logo. In these situations, people can also show compensation behaviour but these are not measured with the present questionnaire.

Furthermore, research has shown that that the concept of 'compensation behaviour' is not always unfavourable in weight balancing. For example, a study from Wammes and colleagues revealed that people use compensation behaviour in reaction to 'overbalanced' energy intakes. After bingeing episodes, people tended to eat healthier foods or tended to have more exercise to compensate for their surplus intake and in doing so thought to avoid gaining weight ${ }^{(20)}$. Further, as mentioned before, Knäuper and collegues developed the CHBS that measures beliefs about how people allow themselves unhealthy behaviour, but compensate for the negative effects and guilt feelings with healthy behaviour afterwards ${ }^{(21)}$. Translating these beliefs properly into behaviour might neutralize the negative effect of unhealthy behaviour. Nevertheless, compensatory beliefs are not always translated adequately ${ }^{(22)}$.

Furthermore, it is important to emphasize that the questionnaire does not explore behaviour, but only beliefs. Beliefs are important to determine behaviour but people have several types of beliefs that counterbalance each other to perform behaviour. In addition to behavioural beliefs which are measured with the Diet-CHBS, people could also have other types of beliefs like normative beliefs or control beliefs. Thus in addition to behavioural beliefs, other beliefs are responsible for performing a particular behaviour ${ }^{(23)}$. Besides beliefs, other determinants can influence people's behaviour. For example, physiological factors like hunger can influence people's compensation behaviour ${ }^{(24)}$. In this way, people could eat more products with a more favourable nutrient composition in addition to small meals. Another explanation could be that for some people eating is habitual and therefore not the result of considered reflection ${ }^{(25)}$. Therefore, it is recommended to test the Diet-CHBS in intervention studies to explore its overlap with actual compensation behaviour.

Another recommendation is to determine Diet-CHBS scores in more representative populations like target groups for nutritional interventions aimed at decreasing energy intake. Furthermore, it is interesting to compare Diet-CHBS scores for several populations. As described earlier, the participants involved in Study 1 were relatively young, well-educated and had a healthy weight. This is also a likely explanation for the relatively low score on the Diet-CHBS and its subscales. A final recommendation is to determine compensation beliefs in a more wide-ranging perspective than for interventions aimed at portion sizes and front-of-package labelling to decrease energy intake.

\section{Conclusions}

The Diet-CHBS is a valid and reliable measure that can help to provide more insight into the beliefs regarding compensatory health beliefs. Whereas the CHBS can be used for a more general assessment of compensatory health beliefs in a broader domain, we suggest using the Diet-CHBS for measuring compensatory health beliefs in the evaluation of nutritional interventions. In doing so, the possible impact of people's diet-related compensatory health beliefs on the main results can be determined.

\section{Acknowledgements}

This research received no specific grant from any funding agency in the public, commercial or not-for-profit sectors. The authors have no conflict of interest to declare. All authors conceived the study idea and design. M.P.P. and W.M.V. collected data, contributed to interpretation of the results and led the writing. I.H.M.S. and E.L.V. provided input and critical feedback on drafts of the manuscript. The authors thank all participants for their contribution in the studies.

\section{References}

1. World Health Organization (2011) Factsheet on obesity and overweight. http://www.who.int/mediacentre/factsheets/ fs311/en/index.html (accessed May 2011).

2. Hill JO, Peters JC, Catenacci VA et al. (2008) International strategies to address obesity. Obes Rev 9, Suppl. 1, 41-47.

3. Herman CP \& Polivy J (2005) Normative influences on food intake. Physiol Behav 86, 762-772.

4. Steenhuis IHM \& Vermeer WM (2009) Portion size: review and framework for interventions. Int J Behav Nutr Phys Act 6, 58.

5. Vyth EL, Steenhuis IHM, Mallant SF et al. (2009) A front-ofpack nutrition logo: a quantitative and qualitative process evaluation in the Netherlands. J Health Commun 14, 631-645.

6. Lioret S, Touvier M, Dubuisson C et al. (2009) Trends in child overweight rates and energy intake in France from 1999 to 2007: relationships with socioeconomic status. Obesity (Silver Spring) 17, 1092-1100.

7. Schokker DF, Visscher TLS, Nooyens ACJ et al. (2007) Prevalence of overweight and obesity in the Netherlands. Obes Rev 8, 101-107.

8. Feunekes GI, Gortemaker IA, Willems AA et al. (2008) Front-of-pack nutrition labelling: testing effectiveness of different nutrition labelling formats front-of-pack in four European countries. Appetite 50, 57-70.

9. Provencher V, Polivy J \& Herman CP (2009) Perceived healthiness of food. If it's healthy, you can eat more! Appetite 52, 340-344.

10. Chandon P \& Wansink B (2007) The biasing health halos of fast-food restaurant health claims: lower calorie estimates and higher side-dish consumption intentions. J Consum Res 34, 301-314.

11. Carels RA, Harper J \& Konrad K (2006) Qualitative perceptions and caloric estimations of healthy and unhealthy foods by behavioral weight loss participants. Appetite $\mathbf{4 6}$, 199-206.

12. Fowler SP, Williams K, Hunt KJ et al. (2005) Diet soft drink consumption is associated with increased incidence of overweight and obesity in the San Antonio Heart Study. Diabetes 54, A258. 
13. Rolls BJ, Roe LS, Halverson KH et al. (2007) Using a smaller plate did not reduce energy intake at meals. Appetite 49, 652-660.

14. Khan U \& Dhar R (2006) Licensing effect in consumer choice. J Mark Res 43, 259-266.

15. Finkelstein SR \& Fishbach A (2010) When healthy food makes you hungry. J Consum Res 37, 357-367.

16. Chiou WB, Yang CC \& Wan CS (2011) Ironic effects of dietary supplementation: illusory invulnerability created by taking dietary supplements licenses health-risk behaviors. Psychol Sci 22, 1081-1086.

17. Knäuper B, Rabiau M, Cohen O et al. (2004) Compensatory health beliefs: scale development and psychometric properties. Psychol Health 19, 607-624.

18. Field A (2005) Discovering Statistics Using SPSS, 2nd ed. London: Sage.

19. Vermeer WM, Steenhuis IH, Leeuwis FH et al. (2011) Small portion sizes in worksite cafeterias: do they help consumers to reduce their food intake? Int J Obes (Lond) 35, 1200-1207.
20. Wammes B, French S \& Brug J (2007) What young Dutch adults say they do to keep from gaining weight: selfreported prevalence of overeating, compensatory behaviours and specific weight control behaviours. Public Health Nutr 10, 790-798.

21. Rabiau M, Knauper B \& Miquelon P (2006) The eternal quest for optimal balance between maximizing pleasure and minimizing harm: the compensatory health beliefs model. Br J Health Psychol 11, 139-153.

22. Kronick I, Auerbach RP, Stich C et al. (2011) Compensatory beliefs and intentions contribute to the prediction of caloric intake in dieters. Appetite 57, 435-438.

23. Ajzen I (1991) The theory of planned behavior. Organ Behav Hum Decis Process 50, 179-211.

24. Story M, Neumark-Sztainer D \& French S (2002) Individual and environmental influences on adolescent eating behaviors. J Am Diet Assoc 102, 3 Suppl., S40-S51.

25. Cohen D \& Farley TA (2008) Eating as an automatic behavior. Prev Chronic Dis 5, A23. 